\title{
Les révélations du "journalisme d'investigation"
}

Monsieur Dominique Marchetti

\section{Citer ce document / Cite this document :}

Marchetti Dominique. Les révélations du "journalisme d'investigation". In: Actes de la recherche en sciences sociales. Vol. 131132, mars 2000. Le journalisme et l'économie. pp. 30-40;

doi : https://doi.org/10.3406/arss.2000.2663

https://www.persee.fr/doc/arss_0335-5322_2000_num_131_1_2663

Fichier pdf généré le 23/04/2018 


\section{Zusammenfassung}

Investigations-Journalismus

Seit den 70er und 80er Jahren haben die nationalen Medien allgemeiner und politischer Information regelmäßig die Verdienste des "Investigations-Journalismus" und seinen Beitrag zur Aufklärung von "Affären" lobend hervorgehoben. Zum Verständnis des Aufkommens dieses neuen Modells professioneller Vortrefflichkeit, welches indes nur einen kleinen Mitgliederteil der Berufssparte beruhrt, ware zu zeigen, dass es sich im wesentlichen zunächst einmal um das Symptom einer Reihe externer Anderungen im Umkreis des journalistischen Feldes handelt, namentlich diejenigen, die den politischen und juristischen Bereich beruhren, und des Zustandes der Beziehungen, die die verschiedenen Räume zueinander unterhalten. Namentlich ware hervorzuheben, in welchem Maß der Medienraum strategisch an Bedeutung gewonnen hat, wohingegen seine Autonomie weiterhin schwach geblieben ist. Er mediatisiert weitgehend das Resultat den verschiedenen sozialen Räumen interner Kräfteverhältnisse, die er sich in seine eigene Sprache zuruckubersetzt. Auf diese Weise beruht der Investigations-Journalismus oft weniger auf journalistischem Recherchieren, als auf Eigenuntersuchungen des Staates in Gestalt amtlicher Berichte, gerichtlicher Ermittlungsverfahren und vergleichbarer Quellen. Aber es kommt dem Artikel auch insbesondere darauf an zu zeigen, wie und warum die Affären nur in dem Maße haben aufkommen können, wie zugleich die externen Veränderungen innerhalb des Feldes der nationalen Medien allgemeiner und politischer Information, und namentlich bei einer Gruppe politisierter Journalisten, die derart ein neues journalistisches Gebiet begrunden, auf ein entsprechendes Echo gestofien sind. Das Entstehen des InvestigationsJournalismus und sein Erfolg im Diskurs des Berufsumkreises ist Ergebnis und Indiz von Veränderungen, die den journalistischen Raum insgesamt beruhrt haben. Er ist zugleich von beruflichem, wie von kommerziellem Belang, insofern aufgrund der Affären eben sowohl der individuelle Ruf (der Journalisten), wie der kollektive (der Medien) auf dem Spiel stehen. Die investigierenden Journalisten haben als Teilhaber einer Entwicklung zur Professionalisierung dazu beigetragen, neue Formen interner Konkurrenz zu schaffen und sichtbar werden zu lassen, die weniger einer politischen, als einer beruflichen Logik folgen, eine Homogenisierung der Praxis verschiedener Bereiche des Pressewesens, und eine professionellere Definition des Berufs, innerhalb welcher, angesichts des Anwachsens okonomischer Zwange, und mehr noch der Spezialisierung der Information, ein neuer Berufstypus, der des selbsternannten „Rächers der Gesellschaft", entsteht. Der Erfolg dieser neuen, dominanten öffentlichkeitsDarstellung beruht zudem weitgehend auf dem diesbezuglich von den Journalisten gegenuber dem Außenfeld gemachten Gebrauch : Sie stellt ein bevorzugtes Vehikel beruflichen Selbstbeweihräucherns dar, weil es geeignet ist, die Legitimität und die soziale Rolle der Journalisten zu einer Zeit öffentlich herauszustellen, wo diese mehr und mehr in das Feuer der Kritik geraten. In der Nachfolge zweier traditioneller Gattungen des französischen Journalismus, dem politischen Journalismus und der juristischen Berichterstattung, hat der Investigations-Journalismus schliefèlich Auswirkungen auf die Informationsbearbeitung selbst gehabt, indem er dazu beitrug, einer kritischeren Konzeption der Behandlung politischer und Informationen des Rechtswesens zum Durchbruch zu verhelfen, die vermischten Meldungen zu politisieren und die politischen Nachrichten in Form vermischter Meldungen zu publizieren.

\section{Résumé}

Les révélations du « journalisme d'investigation »

Depuis les années 70 et 80 , les médias nationaux d'information générale et politique vantent régulièrement les mérites du " journalisme d'investigation » et son rôle dans les " affaires ». Pour comprendre l'émergence de ce nouveau modèle d'excellence professionnelle, bien qu'il ne concerne qu'un faible nombre de professionnels, il faudrait montrer dans quelle mesure il est d'abord largement le symptôme d'une série de changements externes au champ journalistique, notamment ceux qui ont affecté les univers politique et judiciaire, et de l'état des relations que ces différents espaces entretiennent entre eux. II faudrait notamment faire apparaître à quel point l'espace médiatique est devenu stratégique mais demeure très faiblement autonome. Ce qu'il médiatise est largement le produit de rapports de forces internes à différents espaces sociaux qu'il retraduit selon ses logiques propres. Ainsi, le «journalisme d'investigation » repose souvent moins sur des enquêtes journalistiques que sur des enquêtes d'État (rapports, enquêtes judiciaires, etc.). 
mesure où ces transformations externes ont aussi trouvé des échos au sein du champ des médias nationaux d'information générale et politique, notamment auprès d'un groupe de journalistes politisés qui fonde une nouvelle spécialité journalistique. En effet, l'essor du «journalisme d'investigation » et son succès dans les discours professionnels est le produit et le révélateur de transformations qui ont affecté l'espace journalistique. II représente un enjeu autant professionnel que commercial dans la mesure où à travers les « affaires » se jouent des réputations individuelles (les journalistes) et collectives (les médias). Participant d'un mouvement de " professionnalisation », les «journalistes d'investigation» ont contribué à introduire et à révéler de nouvelles formes de concurrences internes, qui obéissent moins à des logiques politiques que professionnelles, une homogénéisation des pratiques entre les différents types de presse et une définition plus professionnelle du métier (faisant émerger une nouvelle figure, celle de « justicier ») face à la montée des contraintes économiques mais plus encore de l'information spécialisée. Le succès de cette nouvelle représentation publique dominante du journalisme doit aussi beaucoup aux usages externes qu'en font les journalistes : elle est un des supports privilégiés d'autocélébration professionnelle qui permet de réaffirmer publiquement la légitimité et le rôle social des journalistes à une période où ils sont de plus en plus critiqués. Enfin, rompant avec deux genres traditionnels du journalisme français, le journalisme politique et la chronique judiciaire, le «journalisme d'investigation » a eu des effets sur le traitement de l'information en contribuant à imposer une conception plus critique du traitement de l'information politique et judiciaire, politisant le fait divers et « fait diversifiant » l'actualité politique.

\section{Abstract}

The revelations of "investigative journalism"

Since the 1970s and 1980s, both the general and the political national newspapers have regularly praised "investigative journalism" and its role in unveiling "scandals". To gain an understanding of how this new model of professional excellence arose, even though it concerns only a small number of journalists, requires showing the extent to which it is first of all largely symptomatic of a series of changes that have occurred outside the field of journalism, especially in the areas of politics and justice, and the state of the relations these spaces entertain with each other. In particular it is important to show that the media space has become highly strategic notwithstanding its relative lack of autonomy. What is mediatized is largely the outcome of power relations within different social spaces that are then translated according to media logics. Thus "investigative journalism" is often based less on journalists investigations than on State-sponsored studies : reports, inquests, etc. The present article endeavors to show how and why these "scandals" have come out only insofar as these external changes have also found an echo in the field of the general and political national papers, in particular with a group of politicized journalists comprising a new journalistic speciality. In effect, the rise of "investigative journalism" and its success in professional discourses results from and reveals transformations affecting the space of journalism. It is both a professional and commercial issue, for scandals both make and break individual and collective reputations : journalists and newspapers. Investigative journalists play a role in the trend towards "professionalization", and have been instrumental in introducing and revealing new forms of internal competition, governed less by political than by professional logic, a homogenization of the practices in various types of newspapers and a more professional definition of the occupation (giving rise to a new figure, the righter of wrongs), in the face of increasing economic pressures but even more of specialized reporting. The success of this new dominant public representation of journalism owes much to the way journalists use it vis-a-vis the outside world : it is one of the favorite supports for professional self-celebration which enables journalists to publicly assert their legitimacy and the social role they play at a time when they are coming under increasing criticism. Lastly, breaking with two traditional genres of French journalism - political journalism and the legal-affairs column - investigative journalism has affected the way news is processed by helping impose a more critical conception of the way political and legal news are handled, politicizing the human-interest story and making political events "humanly interesting".

\section{Resumen}

Las revelaciones del "periodismo de investigación"

Desde las décadas del 70 y el 80 , los medios nacionales de información general y política ponderar regularmente los méritos del "periodismo de investigación", asi como el papel que desempena en los "affaires". No obstante, este nuevo modelo de excelencia profesional sólo atañe a un pequeño número de profesionales. Para comprender su emergencia, habria que mostrar en qué medida es el síntoma de dos fenómenos : por un lado, de una série de cambios producidos en ámbitos externos al campo periodístico, concretamente aquéllos que han afectado al universo de la política y la justicia ; y por el otro, del estado de las relaciones que se establecen entre los diferentes espacios. Habria que demostrar, sobre todo, hasta 
qué punto el espacio mediático se ha tornado estratégico, aunque siga teniendo muy poca autonomía. Cuando el espacio mediático informa acerca del resultado de las relaciones de fuerzas internas que se expresan en los distintos espacios sociales, lo hace interpretándolo según su propio enfoque. Asi pues, el "periodismo de investigación" a menudo se basa más en las investigaciones que emanan del Estado (informes, pesquisas judiciales, etc.) que en las investigaciones periodisticas.

En este articulo se intenta mostrar de qué manera y por qué los "affaires" pueden surgir únicamente si las mencionadas transformaciones externas también tienen eco en el campo de los medios nacionales de información general y política, fundamentalmente en el grupo de periodistas politizados. Es éste el grupo que establece los cimientos de una nueva especialidad periodistica. El auge del "periodismo de investigación", cuya gran aceptación se ve reflejada en el discurso de los profesionales, es el producto que deja traslucir las transformaciones que se han operado en el espacio periodístico. Asimismo, constituye un elemento de capital importancia tanto a nivel profesional como comercial, en tanto y en cuanto a través de los "affaires" se juega la reputación individual (de los periodistas) y la colectiva (de los medios masivos de comunicación). Al participar en el movimiento de "profesionalización", los "periodistas de investigación" han contribuido a introducir nuevas formas de competencia interna y a revelar otras. Todas ellas responden a una lógica de índole más profesional que política. Se observa una homogeneización de las prácticas habituales en los diferentes tipos de prensa, asi como una definición más profesional del periodismo (que hace surgir un nuevo personaje, el del "justiciero") al aumentar las dificultades económicas, pero más aún frente al desarrollo de la información especializada. El éxito de esta nueva representatión pública dominante del periodismo también se explica en gran parte por el modo en que los periodistas la utilizan en otros ámbitos, ya que es uno de los soportes predilectos de autocelebración profesional que permite reafirmar públicamente la legitimidad y la función social de los periodistas en una época en que se los critica cada vez más. Por último, el "periodismo de investigación." ha roto con los dos géneros tradicionales del periodismo francés, el periodismo politico y la crónica judicial. Al contribuir a imponer una conception más critica del tratamiento de la informatión política y judicial, politizando las noticias breves sobre sucesos cotidianos deplorables y "cotidianizando" la actualidad política, el "periodismo de investigación" ha producido consecuencias importantes en la manera de abordar la información. 


\section{Dominique Marchetti}

\section{LES RÉVÉLATIONS DU "JOURNALISME D'INVESTIGATION"}

1 - Cette phrase revient régulièrement dans un débat entre professionnels: "Quel journalisme d'investigation? Table ronde avec Jacques Derogy, Gilles Gaetner, Edwy Plenel et James Sarazin ", in Esprit, $n^{\circ} 167,1990$, p. $18-34$

2 - On peut se reporter à ce sujet à plusieurs ouvrages: J. Derogy et J.-M. Pontaut, Investigation passion. Enquête sur 30 ans d'affaires, Paris, Fayard, 1993; E. Plenel. Un temps de chien, Paris, Stock, 1994 ; D. Robert, Pendant les "affaires", les affaires continuent..., Paris, Stock, 1996

3 - R. Lenoir et S. Poilleux, Justice et médias. Le secret de l'instruction et le droit au respect de la présomption d'innocence, Paris, CREDHESS, universite de Paris-1, mars 1997, chap. v.

4 - 11 conviendrait dès lors de n'utiliser les expressions "affaires " et "journalisme d'investigation " qu'entre guillemets tout au long du texte, mais cela rendrait la lecture difficile. 5 - S'inscrivant dans le prolongement d'un travail de thèse, cet article est un état d'une enquête en cours visant à comparer les logiques à l'cuure dans plusieurs sousespaces spécialisés du champ journalistique.

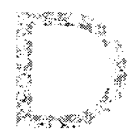

epuis les années 80 , les médias nationaux d'information générale et politique vantent régulièrement les mérites du «journalisme d'investigation » et leur rôle dans les "affaires». Les « unes" annonçant des révélations sur des affaires judiciaires qui impliquent notamment des personnalités politiques de premier plan, sont de plus en plus nombreuses. Beaucoup de journalistes semblent accorder à l'investigation une supériorité évidente sur les autres pratiques journalistiques, rejetant parfois l'expression au prétexte qu'elle serait un pléonasme ${ }^{1}$. La valorisation de l'" enquête » dans les discours des professionnels est liée à l'émergence d'une nouvelle catégorie de journalistes qui doivent leur notoriété aux affaires qu'ils ont "sorties». Un certain nombre d'entre eux publient régulièrement des ouvrages sur les dossiers qu'ils couvrent ${ }^{2}$, ce qui leur vaut d'être invités dans des émissions de télévision. Participant au développement d'un marché éditorial spécifique sur les affaires ${ }^{3}$, ils deviennent des plumes presque aussi sollicitées par les maisons d'édition que les éditorialistes ou les présentateurs vedettes des chaînes de télévision.

On ne peut qu'être surpris par le décalage qui existe entre l'importance publique prise par le journalisme d'investigation en France depuis le début des années 80 et le faible nombre de ceux qui le pratiquent ou prétendent le pratiquer, même s'il est difficile de cerner ce groupe. Le titre même et les frontières de cette population font l'objet de luttes ${ }^{4}$. Mais on peut tout de même tenter de caractériser ce type de journalisme en précisant que ceux qui sont désignés dans le milieu comme des journalistes d'investigation appartiennent généralement à des rédactions de la presse écrite (quotidiens et hebdomadaires nationaux d'informations générales et politiques) et portent souvent le titre de reporter et surtout de grand reporter ou de chef d'enquête. Rattachés à des services d' "Informations générales » ou "Société » ou bien à des cellules, des sections "Enquêtes » ou "Investigations", leur spécialité est de suivre des affaires, c'est-à-dire de s'intéresser à des questions donnant lieu à des poursuites judiciaires ou à des enquêtes des services spécialisés. D'autres, plus marginaux, ont quitté les rédactions et publient leurs enquêtes sous forme d'ouvrages.

Pour comprendre la montée de ce journalisme, c'est-à-dire aussi des affaires dans le champ politique, il faudrait montrer dans quelle mesure il est d'abord largement le symptôme d'une série de changements externes au champ journalistique, notamment ceux qui ont affecté les univers politique et judiciaire, et de l'état des relations que ces différents espaces entretiennent entre eux ${ }^{5}$. En effet, l'essor de cette nouvelle spécialité journalistique est d'abord lié à des transformations affectant le champ politique qu'on ne peut qu'esquisser ici. Il faudrait par exemple détailler comment l'augmentation du coût des activités politiques, le développement de nouvelles techniques (sondage, marketing, communication) ou encore la transformation structurelle de l'action publique, notamment 
avec la décentralisation, ont pu conduire à des pratiques qui ne faisaient pas l'objet de poursuites judiciaires: soit parce qu'elles n'existaient pas auparavant (ou tout du moins pas à grande échelle), soit parce qu'elles étaient perçues comme "normales". Mais c'est aussi ce qu'on pourrait appeler la dépolitisation des enjeux internes au champ politique induite par un "alignement néolibéral ", notamment du Parti socialiste, qui a contribué à modifier les conditions de la lutte politique. L'affaiblissement des oppositions traditionnelles droite/gauche, du fait notamment de l'homogénéisation croissante du personnel politique issu des écoles du pouvoir, a déplacé au moins en partie la lutte politique vers des enjeux plus strictement moraux, la moralité des hommes politiques devenant un des enjeux des conflits internes. La place prise par les affaires à partir des années 80 peut être ainsi rapportée à cette nécessité croissante pour les hommes politiques de se démarquer en utilisant la "moralité » comme arme politique, pour qualifier ou disqualifier, contexte qui n'est pas sans rappeler mutatis mutandis celui de la troisième République. Cette évolution n'est pas spécifiquement française, même si elle peut prendre d'autres formes comme aux États-Unis et en Grande-Bretagne, où c'est davantage la "moralité » touchant à la vie privée qui est mise en cause publiquement.

La médiatisation des affaires renvoie toujours à des faits divers concernant des crimes, qui peuvent d'ailleurs être politisés, ou parfois à des actes terroristes. Or le terme n'exprime plus seulement aujourd'hui ce qui fait l'objet d'une enquête judiciaire mais tout ce qui peut donner lieu à une controverse publique, c'est-à-dire à un débat médiatico-politique. Cette expression renvoie aussi à des affaires dites "politiques " ${ }^{\circ}$. Si celles-ci ne sont bien évidemment pas une nouveauté en France, le terme recouvre en fait des réalités différentes suivant les époques. Philippe Garraud ${ }^{\top}$ explique que, du début de la troisième République aux années 70 , ces affaires sont peu nombreuses, portent sur "des cas d'escroquerie à l'épargne", les hommes politiques y jouant "un rôle secondaire " et étant rarement sanctionnés. Alors qu'à partir du milieu des années 80 , il s'agit d' "affaires politico-financières" plus importantes en nombre, qui concernent des "cas denrichissement personnel " ou de "financement occulte des activités politiques et partisanes", touchent "toutes les catégories de personnel et d'institutions politiques» et débouchent sur des condamnations judiciaires. Par exemple, plusieurs ministres, députés ou maires ont dû démissionner ou ont quitté l'activité politique à la suite d'affaires judiciaires. Depuis le début de la décennie 80 , les affaires ${ }^{8}$ sont effectivement liées à des activités plus directement politiques (les affaires politicofinancieres: l' "affaire Urba", "MNEF", "Elf», "Carignon ", "Noir", "Longuet "), mais aussi à des faits divers politiques ("affaire des Irlandais de Vincennes", "Greenpeace", "Carrefour du développement", «affaire Tiberi »), qui, du seul fait de la notoriété des protagonistes ou des services de l'État engagés (les services de renseignements, une cellule de l'Élysée, des ministères, des amis du président de la République), peuvent constituer un enjeu politique, ou encore des affaires touchant à des secteurs qui ont partie liée avec l'État comme l'économie ("affaire Pechiney», "affaire du Crédit lyonnais") ou la science (l' "affaire du sang contaminé ", l' "affaire de la vache folle", l' "affaire de l'amiante"). Ce sont donc au sens large les pratiques du pouvoir d'État ou en lien avec lui dans le cas, par exemple, des entreprises qui ont des marchés publics, qui sont visées.

La montée des affaires et du journalisme d'investigation a été rendue possible dans la mesure où ces luttes de concurrence au sein du champ politique ont rencontré des transformations à la fois dans les champs judiciaire et médiatique, le premier permettant la sortie des affaires, le second contribuant à leur donner un écho public auprès des électeurs. Au sein de l'univers judiciaire, des nouveaux agents étaient particulièrement disposés à contribuer par leurs enquêtes à dévoiler des affaires politiques. En effet, le recrutement dans le champ judiciaire est marqué, à la fin des années 60, par l'arrivée de nouvelles générations de juges d'instruction (les juges « rouges"), qui sont plus souvent issus des classes moyennes et plus sensibles aux droits de l'homme, porteurs de dispositions "soixante-huitardes». Ainsi, le Syndicat de la magistrature, créé à cette époque, participe au développement de nouvelles pratiques et de représentations du milieu ${ }^{9}$, destinées à affirmer une plus grande autonomie du champ judiciaire à l'égard du champ politique, dénonçant par exemple la mainmise du pouvoir politique sur la justice à travers notamment les nominations et le déroulement des carrières. Ce groupe, suivi par d'autres dans les années 80 , a fortement contribué à la montée en puissance du droit pénal, à la valorisation croissante des juges, redéfinissant ainsi leur identité sociale et professionnelle ${ }^{10}$, mais aussi à la politisation de la vie judiciaire.

6 - Pour une illustration, voir deux numéros spéciaux consacrés aux affaires: Les Cahiers de L'Express, juillet 1991 et L'Evenement du jeudi, 30 janvier-5 février 1992

7 - P. Garraud, "Les nouveaux juges du politique en France ", in Critique internationale, $\mathrm{n}^{\circ} 3,1999, \mathrm{p} .125-139$

8 - Sur certaines affaires, on peut se reporter aux analyses de P. Lascoumes, Corruptions, Paris, Presses de Sciences po, 1999

9 - R. Lenoir, "La parole est aux juges. Crise de la magistrature et champ journalistique", in Actes de la recherche en sciences sociales. $\mathrm{n}^{\circ} 101-102,1994$, p. 77-84

10 - Voir V. Roussel, "Les magistrats dans les scandales politiques". in Revue française de science politique, vol. $48, n^{\circ} 2.1998, p .245-273$. 


\section{Un espace stratégique}

Si les affaires et l'investigation ont pris une telle place, c'est également parce qu'elles sont le produit et le révélateur de la position stratégique qu'occupent désormais les médias dans les luttes internes au champ politique ${ }^{11}$ et au champ judiciaire et dans les relations qu'ils entretiennent entre eux. Le champ journalistique est stratégique non pas parce qu'il représenterait un "quatrième pouvoir", mais parce qu'il permet d'agir par ricochet sur différents univers sociaux. Comme le montrent les affaires, ce que médiatise l'espace journalistique est en partie le produit de rapports de forces internes à différents espaces sociaux qu'il retraduit selon sa logique propre. Ce ne sont donc pas seulement des facteurs économiques qui limitent l'investigation journalistique, tels que la crainte des procès ou encore le manque de temps (voir encadré p. 36), mais aussi la dépendance à l'égard des champs politique et judiciaire, qui détiennent le monopole des informations en ce domaine.

La manière de présenter l'investigation comme une enquête journalistique de longue durée, consistant à «aller chercher» l'information, voire à la voler, masque et reflète des réalités bien connues des professionnels eux-mêmes, qu'ils évoquent très souvent en privé, notamment de manière humoristique. Comme les affaires dites «du sang contaminé " ${ }^{12}$ ou de la "Commission européenne ${ }^{13}$ le font apparaître, les divisions des journalistes reproduisent très souvent en partie les divisions des champs qu'ils couvrent. Ceux qui «sortent » des affaires s'appuient toujours sur différents types d'agents - qui varient selon les cas -, que ce soit des magistrats, des avocats, des policiers ou des gendarmes, des hommes politiques ou leur entourage, d'anciens employés d'une entreprise ou d'un service politique ou administratif, de membres d'associations ou de comités de soutien, etc. L'accès au dossier se fait souvent à travers une alliance objective avec l'une des parties ou plus rarement avec un magistrat, un policier ou un gendarme. Les intérêts des journalistes d'investigation ont rencontré et rencontrent ceux d'autres agents pour lesquels la presse est parfois considérée comme un recours pour agir sur une instruction judiciaire, ou plus largement sur les autorités judiciaires et politiques. Il n'est pas rare que les journalistes fonctionnent, comme le disent certains d'entre eux, comme des «boîtes aux lettres": ils reçoivent des documents ou on leur suggère d'aller enquêter sur tel ou tel fait, sélectionnant ce qu'ils doivent diffuser ou pas.

En fait, l'investigation renvoie moins (à quelques rares exceptions près) à des enquêtes proprement journalistiques qu'à la capacité de se procurer avant les concurrents des éléments concernant des enquêtes judiciaires classiques (annonces exclusives de mises en examen, de plaintes, de perquisitions, divulgations de pièces du dossier, déclarations à la presse, etc.) et les rapports administratifs (voire les prérapports) commandés sur des sujets sensibles. Paradoxalement, ce journalisme d'humeur anti-institutionnelle s'appuie, pour critiquer des structures publiques et leurs tutelles politiques, sur des enquêtes de l'État qui sont communiquées en avant-première. Les fuites sont orchestrées par les membres de cabinets ministériels, les membres de commission ad hoc ou les rapporteurs, voire par le principal responsable de telle administration. Ainsi, les rapports de l'Inspection générale des affaires sociales (IGAS), voués à l'origine à rester internes, sont rendus publics et reçoivent une publicité médiatique plus importante à partir de la fin des années 80 et du début des années 90 . Mais plus largement, nombre de rapports administratifs sont l'objet d'une concurrence pour la priorité - et aussi d'une lutte d'interprétations - entre quotidiens et entre quotidiens et hebdomadaires. Qu'une enquête journalistique déclenche une instruction judiciaire (ou un supplément d'enquête) ou administrative, comme ce fut le cas pour l'" affaire du sang contaminé » et qu'un journaliste donne des éléments qui alimentent le dossier d'un magistrat demeure donc une configuration très exceptionnelle. Ce type d'événements fait voir que le champ journalistique est très peu autonome à l'égard d'autres univers sociaux, économique, judiciaire et politique notamment.

\section{Des transformations internes}

Toutefois, les affaires et l'investigation n'ont pu émerger que dans la mesure où ces transformations externes ont aussi trouvé un écho au sein du champ journalistique. En effet, pour comprendre ce qu'est le journalisme d'investigation, dont les défenseurs donnent souvent une image idéalisée ${ }^{14}$, et pour rendre compte du succès qu'il rencontre dans les discours professionnels aujourd'hui, il faut analyser en quoi il est le pro-

11 - Voir P. Champagne, Faire l'opinion. Le nouvau jeu politique, Paris, Minuit, 1991

12 - Voir D. Marchetti. "Contribution a une sociologie des transformations du champ journalistique dans les annees 80 et 90 . A propos d'evenements sida" et du "scandale du sang contamine" ", Paris, doctorat de sociologie sous la direction de P. Bourdieu. Ecole des hautes etudes en sciences sociales, decembre 1997.

13 - D. Georgakakis, "Juger la Commission européenne. Scandale et lournant institutionnel (oclobre 1998-mars 1999)". communication au sixieme congres de l'Association françalse de science politique a Rennes, table ronde $n^{\circ} 2$. septembre-octobre 1999.

14 - Pour une illustration. on peut se reporter a louvrage d'un écrivain-journaliste americain M. Hunter, Le Journalisme d'investigation. Paris, PUF, coll. "Que sais-je?", 1997. 
duit et le révélateur de transformations du champ des médias nationaux d'information générale et politique depuis une vingtaine d'années, notamment parce qu'il représente un enjeu professionnel important.

Les luttes à l'intérieur des champs politique, judiciaire et entre ces champs ont rencontré les intérêts d'un petit groupe de journalistes en formation, qui fonde en France une nouvelle spécialité journalistique $^{15}$. Réinventant l'enquête à la manière des reporters de la fin du XIX siècle, qui avaient adapté des techniques du journalisme américain ${ }^{16}$, les journalistes qui suivent les affaires judiciaires importent le concept de journalisme d'investigation dans le domaine politique. Le Canard enchainé notamment développe des enquêtes dans ce domaine. Spécialisé dans la satire politique, il se tourne davantage, dans les années 50 et 60 , vers l'information et l'investigation ${ }^{17}$. Les guerres d'Indochine et d'Algérie, mais aussi les scandales immobiliers du début de la cinquième République fournissent des sujets d'enquête. L'arrivée, au début des années 70, de Claude Angeli, qui a travaillé dans les rubriques de politique intérieure de plusieurs publications de gauche (Le Nouvel Observateur, Politique hebdo), accentue ce changement éditorial. Mais il faudra attendre les années 80 et 90 pour que les scandales et affaires hebdomadaires du Canard soient repris plus régulièrement par ses confrères (sous forme d'articles ou de citations dans les revues de presse). En effet, l'investigation n'est pas sans susciter de fortes critiques à l'époque ${ }^{18}$ au pôle intellectuel du champ journalistique, notamment dans les quotidiens et les newsmagazines. Au-delà du Canard enchainé, quelques "francs-tireurs" (comme Jacques Derogy à L'Express ou Pierre Péan dans plusieurs rédactions) des services "informations générales" ou "société " s'intéressent aux affaires.

Il est aussi évident que l'exemple américain de l'affaire du Watergate est pour une part dans la consécration de l'investigative reporting. En effet, l'investigation doit beaucoup à l'importation de pratiques qui sont d'abord apparues aux États-Unis et dont Michael Schudson, étudiant l'impact du Watergate sur le journalisme américain ${ }^{19}$, a montré qu'elles avaient davantage à voir avec le pouvoir de ce qu'il appelle un «mythe " qu'avec des changements dans les pratiques journalistiques qui ont suivi l'affaire.

Cette première génération des journalistes d'investigation occupe une position homologue à celle des «juges rouges" des années 70 ou des nouveaux entrants dans le champ judiciaire des années 80 . Ils sont d'autant plus disposés à avoir un rapport critique au pouvoir politique, ou plus largement une humeur anti-institutionnelle, que nombre d'entre eux ont un passé politique de gauche ou ont été politisés très jeunes, témoignant, au détour de certains de leurs ouvrages écrits à la première personne, de leur déception à l'égard de la politique (plus particulièrement de la gauche au pouvoir ${ }^{20}$. Il faudrait montrer plus précisément que la plupart ont eu un rapport de plus en plus critique à la politique bien que les plus jeunes, entrés à la fin des années 80 et au début des années 90 , présentent sans doute un autre profil: de Jacques Derogy, ancien journaliste du Libération d'après-guerre et militant du Parti communiste dont il a été exclu, à Claude Angeli, qui a eu des engagements politiques (il a participé notamment à la création du Secours Rouge en juin 1970) et des responsabilités éditoriales dans plusieurs journaux de gauche, en passant par Edwy Plenel, ancien militant trotskiste et rédacteur de Rouge, journal de la Ligue communiste révolutionnaire, ou encore Pierre Péan, qui s'engage contre l'Algérie française. Au-delà de cette connaissance pratique plus ou moins développée de la politique, ils avaient également, pour certains, un capital scolaire supérieur à la majorité des confrères de leur génération. S'ils ne semblent pas en majorité être titulaires de diplôme d'écoles professionnelles, beaucoup ont suivi des études supérieures à l'université ou dans des grandes écoles (Institut d'études politiques de Paris et province ou École normale supérieure). Leurs cursus correspondent à ceux de leurs confrères de la presse nationale d'information générale: la philosophie, le droit et la science politique pour beaucoup. Ils semblent majoritairement issus des classes moyennes et supérieures : ils sont notamment fils de journalistes, de professions libérales ou de fonctionnaires de catégorie $\mathrm{A}^{21}$.

15 - Pour une histoire du "journalisme dinvestigation " en France voir S. Gerbaud, "Le journalisme d'investigation en France de 1945 a nos jours", Paris, these de doctorat d'histoire, universite Paris-X. 1993.

16 - Dés le début du $x x^{*}$ siècle aux États-Unis, certains phénomènes de corruption politique faisaient déja l'objet d'enquêtes journalistiques agressives (leurs auteurs étant souvent appelés muckrakers, cest-a-dire des "remueurs de boue" ou des "fouille-merde") qui susciterent alors des critiques.

17 - P. Champagne, "Le Canard enchainé, de la satire politique a la défense de la morale publique ", in Liber, n 7,1991, p. 6-8.

18 - Pour une illustration, voir V. Gaglione. L'Influence du journalisme d'investigation du Canard enchainé sur Le Monde : mutation ét dérive du quotidién de référence. Paris, mémoire de maitrise d'information et de communication, option journalisme, universite Paris-Sorbonne, CELSA, 1989, p. 33

19 - M. Schudson. The Power of News, Cambridge, Mass., Harvard University Press, 1995, p. 148.

20 - Pour des illustrations, voir D. Robert, ap. cit. p. 60, ct les interventions de E. Plenel dans: "Quel journalisme d'investigation? ", art cil., p. $18-34$

21 - En l'etat actuel de notre enquéte, il est difficile de préciser davantage les proprietes sociales de ces journalistes, et donc de voir en quoi elles nous permettratent de mieux comprendre notamment ce rapport a la politique et au journalisme politique, et en quoi ils se différencient, sous ce rapport, de certains de leurs confreres. 


\section{Un enjeu commercial et professionnel}

L'importance de la figure du journaliste d'investigation est aussi liée à l'enjeu professionnel qu'il représente depuis le début des années 80 au sein du champ des médias nationaux. De même qu'au tournant du siècle, le grand reportage était un objet de concurrence très fort entre les titres, un support d'autopromotion d'une partie de la presse ${ }^{22}$, «faire de l'investigation » devient pour quelques médias une nécessité professionnelle. Les quotidiens nationaux d'information générale et politique, notamment Le Monde qui a contribué à consacrer le genre en le reprenant à son compte, et l'ensemble des newsmagazines font de ce nouveau produit journalistique une arme dans la concurrence. C'est dans la première moitié des années 80 que les premiers services ou cellules dit(e)s d'investigation(s) ou enquête(s) sont créé(e)s dans plusieurs rédactions de médias nationaux d'information générale et politique. Ce modèle se diffuse au-delà de la presse écrite puisque aujourd'hui les stations de radio et surtout les chaînes de télévision font à leur tour des enquêtes avec des programmes comme Envoyé spécial sur Antenne 2 , puis France 2, Le Droit de savoir sur TFl, Capital ou Hors Stade sur M6 et plus encore Le Vrai Journal, dont les responsables souhaiteraient qu'il devînt l'équivalent du Canard enchaîné à la télévision, ou encore de 90 Minutes diffusés sur Canal Plus. Cette promotion de l'investigation s'étend aussi à différentes spécialités comme la science et la médecine, le sport, etc.

La constitution de cette nouvelle spécialité journalistique est le produit et le révélateur d'une transformation de la structure de cet espace, du fait de la montée des contraintes économiques. Les médias audiovisuels (et particulièrement la télévision à partir des années 70-80), vers lesquels se tourne un public de plus en plus large et qui attirent les publicitaires, sont devenus le pôle autour duquel s'organise en partie le champ journalistique. Leur développement a renforcé le pôle commercial et, du même coup, homogénéisé la production de l'information. Les radios et les télévisions ont aussi contribué à intensifier la concurrence économique au sein de la presse écrite généraliste, obligeant les différents supports à marquer leurs différences par des "révélations » ou à trouver des nouveaux sujets.

La presse quotidienne française est touchée, depuis les années 50, par la baisse de la diffusion qui se confirme surtout pour les titres nationaux d'information générale et politique : en 1994, ils avaient un tirage total de 1,3 million ${ }^{23}$ contre 2,9 en $1980,4,28$ en 1970 et 5,95 millions d'exemplaires en $1946^{24}$. Cette tendance concerne tout particulièrement la presse d'opinion ${ }^{25}$ : le nombre de quotidiens nationaux passe de 26 en juin 1945 à 16 en juin $1950^{26}$. Cette évolution se confirme ensuite, notamment dans les décennies 80-90, avec la disparition de deux titres marqués politiquement: Le Matin de Paris, classé à gauche, et Le Quotidien de Paris, classé à droite. D'autre part, si Le Figaro maintient une diffusion totale payée ${ }^{27}$ légèrement en hausse $(300251$ en 1980 à 360441 en $1998^{28}$ ), L'Humanité (138 731 exemplaires en 1980 contre 53521 en 1998) continue à perdre, sur la période, des lecteurs. La presse quotidienne populaire conserve un public populaire, mais elle ne se distingue plus par l'ampleur de ses ventes qui ont sensiblement diminué. Ainsi, France Soir, alors qu'il vendait près de 1 million d'exemplaires en 1964 contre 700000 en 1976, a continué à chuter fortement dans les années 80 et 90 : sa diffusion, qui était de 403085 en 1980, est passée en dessous des 200000 exemplaires en 1994, pour atteindre 156106 exemplaires en 1998. Malgré la forte progression du Parisien (qui strictement régional à l'origine est devenu national avec son édition Aujourd'hui) dans les années 80 et 90 (475 417 en 1998 contre 333971 exemplaires en 1980), le pôle populaire de la presse quotidienne nationale est très affaibli. Dans le secteur des newsmagazines, qui a poursuivi sa progression en termes de diffusion totale payée représentant 1,8 million de lecteurs en 1998 (pour 6 titres) contre 1,17 en 1983 (pour 3 titres), l'arrivée de L'Événement du jeudi, à partir de 1984, puis de Marianne en 1997, a contribué à renforcer cette concurrence économique qui s'est exacerbée, comme le montrent de nombreux indices: le développement des pages de publicité, le lancement de nouvelles formules, la distribution de cadeaux pour les abonnés, les changements de prix, de dirigeants et d'actionnaires majoritaires (rachat de L'Express, du Point et de L'Événement du jeudi). Mais les effets sont encore plus visibles sur le traitement même de l'information, comme le montrent les stratégies marketing dans les titres de "unes", portant désormais moins sur des sujets de politique intérieure ou étrangère que sur des domaines susceptibles d'intéresser un large public : les "problèmes de société ", la santé, le sexe, l'économie pratique (la consommation, l'épargne, les salaires), etc.

Les affaires, parce qu'elles peuvent susciter des ventes supplémentaires ou en tout cas favoriser cette croyance, deviennent donc un enjeu commercial majeur pour ce type de publications. En dépit de déclarations contraires ( On a sorti comme on dit de

22 - Voir C. Delporte, Les Journalistes en France 1880-1950. Naissance et construction d'une profession, Paris, Le Seuil, 1999, p. 70.

23 - Chiffres cités par D. Junqua, "Les quotidiens nationaux français en quête de relance ", in Le Monde diplomatique, avril 1996, p. 26-27. 24 - Source: Tableaux statistiques de la presse, 1987-1988, Paris, SJT1La Documentation française, 1990, p. 111.

25 - C. Bellanger, C. Levy, H. Michel et F. Terrou (sous la dir. de) Histoire générale de la presse française, T. IV: De 1940 à 1958, Paris,

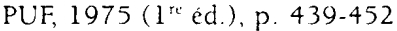

26 - Source: Tableaux statistiques de la presse..., op. cit., p. 111. 27 - Selon la définition de Diffusion contróle, la diffusion totale payee en France et à l'étranger est la somme des abonnements, des ventes au numéro, de la diffusion individuelle par portage el de la diffusion différée payée.

28 - Source: Diffusion contrôle. 
grosses affaires, il n'y a pas d'exemple où ça fait vendre », affirmait en 1994 Jean-François Kahn, alors directeur de la rédaction de L'Événément du jeudi) ${ }^{24}$, tout laisse à penser que les succès de vente imputés à la révélation d' "affaires", dans le cas du Canard enchaîné dès le début des années 70 et du Monde à partir de la première moitié des années 80 , n'ont probablement pas été sans favoriser les "unes " sur ce type de sujet.

\section{Nouvelles formes de la concurrence et homogénéisation des pratiques}

Néanmoins l'investigation est le produit et le révélateur d'une concurrence autant commerciale que professionnelle, ces deux dimensions étant fortement liées. Dans ce cas, la production de l'information répond à une logique beaucoup plus interne qu'externe. Ce type de concurrence, invisible pour le public et, du coup, sous-estimée, est très importante pour les professionnels. Si les affaires font courir certains journalistes, c'est parce que se jouent, à travers elles, les réputations inséparablement collectives (les médias) et individuelles (les journalistes) et le capital de relations avec les «sources» qu'ils peuvent mobiliser. Les cas très liés d'Edwy Plenel et du Monde, à propos de l'«affaire Greenpeace » notamment, le journaliste, s'appuyant sur la réputation de son titre ${ }^{30}$ et inversement, illustrent bien cette accumulation progressive d'un crédit professionnel ou de profits internes ${ }^{31}$. En fait, le journalisme d'investigation, qui participe plus largement d'un mouvement de "professionnalisation ${ }^{32}$, a contribué à la fois à introduire et à révéler de nouvelles formes de concurrences internes entre les différents supports et a tenté d'imposer une définition plus professionnelle du métier.

En effet, dans des états antérieurs du champ journalistique, notamment dans les années 70 , les débats portaient davantage sur les prises de position politiques des différents supports (Le Monde et Le Figaro, Le Monde et L'Humanité, Le Monde et Le Nouvel Observateur) ${ }^{33}$ à l'occasion de tel ou tel événement. Avec le déclin de la presse politique - les journaux se veulent des journaux de journalistes et non de militants -, les enjeux ont pris une dimension plus professionnelle (et plus morale aussi), même si les logiques politiques demeurent toujours présentes. Pour les affaires, comme pour d'autres événements, la concurrence entre les titres prend la forme - elle n'est pas nouvelle mais est, dans ce cas, exacerbée - de la concurrence pour la priorité. Par exemple, à l'occasion des affaires, l'attribution de ce qu'on pourrait appeler la paternité de l'affaire (qui l'a révélée?) est un enjeu primordial.
Cette recherche de l'exclusivité ne s'exprime pas de la même manière dans tous les sous-espaces du champ journalistique. Dans le sport, le cinéma ou la musique par exemple, elle fait l'objet de transactions économiques: les organisateurs de spectacles font systématiquement payer l'exclusivité des images des manifestations les plus importantes. La passation de contrats, qui visent pour les télévisions et les radios à garantir l'exclusivité d'une interview (de photos ou encore d'articles) d'un acteur de cinéma, d'un héros de l'actualité, d'un sportif de haut niveau, etc., tend à être de moins en moins exceptionnelle. Dans dautres secteurs stratégiques (les faits divers, les catastrophes naturelles, mais aussi les affaires), si elle ne prend pas complètement cette forme, la concurrence pour la priorité peut s'en approcher parfois par l'achat d'images, tournées par des amateurs, et d'informations auprès de protagonistes ou de leurs avocats par exemple ${ }^{3+}$. À l'inverse, dans d'autres sous-espaces du journalisme, la concurrence pour la priorité semble moins intense (la rubrique "Social " ${ }^{35}$ ou «Éducation $\left.{ }^{36}\right)$, voire quasi inexistante dans le cas de la chronique judiciaire (voir encadré p. 39).

Mais le second changement lié au journalisme d'investigation, dans la mesure où il produit des "révélations", tient au fait que cette concurrence se traduit par une recherche désormais explicite des "reprises ${ }^{37}$ par les confrères, phénomène qui est aujourd'hui institutionnalisé dans certaines rédactions. Il n'est donc pas anodin que les dirigeants de certains médias développent à présent une sorte de "service après-vente » ou de "service de presse", tout particulierement dans le cas des révélations sur les affaires, pour bénéficier de publicité gratuite, autrement dit d'un impact maximal

29 - Propos extraits de l'emission li' Cercle de minuit, diffusee sur France 2 le 6 février 1994

30 - Voir à ce sujet linterview de E. Plenel parue dans la revue Autrement (n 94-95, 1997): "Ma premiere arme, c'cst mon appartenance au Monde."

31 - A. Accardo, G. Abou, G. Balbastre et D. Marine: Joumalistes au quotidien. Outils pour une socioanalyse des pratiques journalistiques, Bordeaux, Le Mascare1, 1995, p. 46-47

32 - D. Ruellan, Les "pro" du journalisme. De l'etat au statut, la construction d'un espace professionnel, Rennes, PUR, 1997

33 - Pour quelques exemples. voir J. Planchais. Ln hommi du Monde. Paris, Calmann-Levy, 1989 , chap. 15

$3+$ - Plusieurs temoignages de journalistes ou dinformateurs laissen penser que ces deux situations se sont parfois produites dans le scandale du sang contaminé. Pour des exemples sur une autre affaire, voir L. Lacour. Le Bucher de's innocents. Laffaire Villemin, coulisse's, portraits, preuves, engrenages, correspondances, choses vues... Paris, Plon, 1993 35 - 5. Levêque. "La construction journalistique d'une categorie du débat public. Specialisation journalistique et mise en forme du social". Paris, these de science politique sous la direction de M. Offerle. universite Paris-1, 1996

36 - Voir J.-G. Padioleau, "Svstemes d'interaction et rhetoriques journalistiques", in Sociologie du trasail, n= 3, 1976, p. 256-282

37 - Voir D. Marchetti. op. cit. $2^{\circ}$ partie. chap. 1 


\section{Des conditions de production ordinaires}

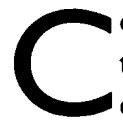
ontrairement aux représentations idéalisées du journalisme d'investigation, les conditions d'urgence dans lesquelles sont placés les journalistes qui traitent des affaires sont relativement semblables à celles de leurs confrères. Loin de mener des enquêtes sur plusieurs mois ou plusieurs années, comme c'est le cas de quelques journalistes-écrivains (par exemple de Pierre Péan ou de Laurence Lacour), les journalistes dits « d'investigation » des quotidiens et des newsmagazines passent beaucoup moins de temps que leurs aînés sur les enquêtes. L'émergence du journalisme d'investigation montre à quel point le rythme de production de l'information s'est accéléré. Les médias audiovisuels ont imposé leur propre temporalité à l'ensemble des autres supports, le culte du direct ayant modifié la temporalité de la presse écrite. Celle-ci vise à concurren- cer en retour les médias audiovisuels, notamment au moyen d'informations en continu (on parle de «feuilletons»). La nécessité de produire régulièrement sous peine de ne pas être rentable ${ }^{a}$, de travailler sur plusieurs affaires simultanément et surtout d'être les premiers face à la concurrence sont autant de facteurs qui expliquent ce nouveau tempo. Et il n'est pas rare que les plus anciens dénoncent ce qu'ils jugent comme des dérives de certains de leurs jeunes confrères. «II y a quelques années, les journalistes étaient loyaux entre eux; aujourd'hui, ils se livrent à une course abominable. L'investigation se réduit à une affaire de vitesse. La peur de voir le concurrent aller plus vite pousse à sortir n'importe quoi. S'il est indispensable d'avoir de l'audace, il ne faut pas oublier la patience : l'investigation, c'est précisément le contraire de la vitesse $»$, expliquait Jacques Derogy ${ }^{b}$. Même si ces conditions ne sont pas sans provoquer des erreurs factuelles ou amènent des journalistes à tirer des conclusions hâtives à partir d'éléments sommaires, certains journalistes $y$ voient au contraire une sorte de progrès puisque, d'une part, nombreux sont ceux qui disent toujours demander avant publication l'avis de toutes les parties, respectant ainsi «l'objectivité journalistique $»$, et, d'autre part, que ce type d'information peut - ou vise seulement à - susciter des réactions ou un débat pouvant « éclairer sous un jour nouveau certains aspects du dossier $»$.

a - Voir sur ce point l'expérience de D. Robert. ancien enquêteur à Libération, Pendant les " affaires ", les affaires continuent..., op. cit., p. 152-153.

b - L'Événement du jeudi, 16-22 octobre 1997. dans les autres médias, tout particulièrement auprès de l'AFP et des chaînes de télévision. C'est la puissance d'amplification des médias audiovisuels, qui conduit des journalistes de la presse écrite à chercher plus ou moins consciemment les reprises par leurs confrères de télévision ou de radio, voire à susciter des sujets d'émissions. Ces reprises sont valorisantes pour le journaliste parce qu'elles donnent du crédit à la fois à son information, à son support et à lui-même ; elles sont appréciées par les supérieurs hiérarchiques : «Au journal, ça leur plaît bien [...] les directeurs sont toujours contents quand ils voient $N$ [nom d'un quotidien national] apparaître à la télé », explique un journaliste d'un grand quotidien national (entretien, 1993). Envoyer des fax ou passer des coups de téléphone aux confrères amis pour annoncer certains scoops est devenu un réflexe dans de nombreuses rédactions. C'est le cas notamment du Monde qui, au-delà des publicités payantes à la radio, développe des stratégies visant à susciter des reprises de ses articles, ce qui ne va pas parfois sans provoquer l'ironie de ses confrères: «On en a marre de faire le service après-vente du Monde », explique un journaliste d'une radio d'information en continu (entretien, 1998).

Le journalisme d'investigation produit et révèle également une homogénéisation des pratiques profes- sionnelles qui n'est pas spécifiquement française, comme l'ont montré des travaux anglo-saxons ${ }^{38}$. Celles qui étaient autrefois l'apanage de la presse dite "populaire " se sont diffusées au pôle intellectuel du champ des médias omnibus nationaux et inversement. Les affaires constituent pour la presse dite "sérieuse » l'équivalent des faits divers dans la presse populaire. Par exemple, les titres de plus en plus " accrocheurs » d'un journal comme Le Monde ressemblent à ceux des newsmagazines ou des quotidiens populaires. Plus largement, c'est souvent la forme ou la présentation des articles et des reportages qui laisse penser à une investigation. Certains journalistes prétendant faire de l'investigation (ou leurs rédacteurs en chef qui rédigent des titres et des " chapeaux » de " unes » notamment) jouent en effet très souvent sur les mots ou les expressions qui pourraient faire croire à une investigation poussée: "Un document inédit ", "une note que s'est procurée $N$ [nom d'un quotidien parisien]", "nouveaux documents sur», "nouvelles pièces au

38 - Voir l'étude comparative de F. Esser, "Tabloidization" of news. A comparative analysis of Anglo-American and German press journalism ", in European Journal of Communication, vol. 14, 3, 1999, p. 291 324 . 
dossier ", "nos révélations", etc. Les pratiques des différents types de journalistes, qui consistent à gonfler les révélations, à ressortir des documents pour être présents sur un sujet ou à les écouler au fur et à mesure pour "faire plusieurs coups", se répandent el suscitent (en privé seulement) l'ironie des uns et des autres.

\section{L'honneur de la profession}

Si le journalisme d'investigation apparaît aussi important dans le milieu, c'est parce qu'il est l'objet de luttes symboliques portant sur la définition même de l'activité journalistique. Aujourd'hui il ne s'agit plus seulement de lutter contre les amateurs, mais de contester certains professionnels (au-delà des traditionnels mauvais exemples : paparazzis ou journalistes corrompus) qui sont censés menacer l'honneur de la profession. Ces enjeux de définition se sont exacerbés à partir des années 80 avec le développement des médias audiovisuels et la montée du journalisme spécialisé, du fait de l'accroissement du nombre d'activités couvertes aujourd'hui par les médias. En disant que l'expression même de "journalisme d'investigation » est un pléonasme, les promoteurs du journalisme d'enquête défendent une conception plus professionnelle et plus autonome du métier, c'est-à-dire, au moins à leurs yeux, plus subversive et plus morale. Le fait d'accoler au mot journalisme non plus un secteur d'activité (politique, scientifique, judiciaire, etc.) ou bien même un média (radio, télévision, presse écrite), mais une méthode de travail en témoigne.

La constitution de ces nouvelles positions peut être en partie comprise comme une réponse des titres de la presse écrite généraliste nationale aux chaines de télévision et de radio venues menacer leur position dominante dans la production de l'information. Elle est devenue une des armes dans la critique interne des "dérives" du journalisme, qui est très souvent une critique des pratiques des journalistes de télévision. Cette affirmation d'un nouvel idéal professionnel s'inscrit plus fortement encore contre le développement des pratiques de communication de plus en plus sophistiquées dans de nombreux secteurs d'activité - que les "enquêteurs" eux-mêmes relaient -, tout particulièrement dans le champ politique. Au « journalisme de connivence ", " de complaisance " ou au « journalisme conventionnel ", « institutionnel ", les journalistes d'investigation opposent une nouvelle définition de l'activité journalistique : "Restaurer l'information dissidente contre le fait accompli, la liberté indocile de la première contre la douce dictature du second. Restaurer la nouvelle qui fait sens contre le communiqué qui fait silence. La révélation qui dérange contre la communication qui arrange ", explique ainsi Edwy Plenel ${ }^{39}$.
C'est pourquoi les journalistes spécialisés (parfois appelés dans un sens péjoratif les "rubricards " ou les "spécialistes») sont souvent cités à titre de mauvais exemples. L'opposition entre les "généralistes » et les «spécialistes », entre les compétences professionnelles générales et la connaissance des sujets traités structure l'ensemble du champ comme le montrent, pour ne prendre que cet exemple, les modes de recrutement et les trajectoires professionnelles des journalistes des médias nationaux d'information générale et politique $^{\text {to }}$. Les journalistes les plus généralistes estiment qu'ils n'ont pas nécessairement besoin de connaître $a$ priori la matière dont ils traitent, mettant en avant la seule maîtrise des techniques journalistiques: disponibilité, débrouillardise, rapidité, culot, capacité à être le premier, indépendance à l'égard des sources, etc. Se posant en observateurs neutres, c'est-à-dire, de ce fait, plus à même d'expliquer clairement les sujets traités aux publics auxquels ils s'adressent, ils s'opposent à ceux qui auraient une vision étroite, partielle, partiale ou trop technique: les journalistes spécialisés souligneraient la continuité plutôt que les nouveautés spectaculaires et auraient des "réflexes corporatistes" de défense du milieu qu'ils " couvrent».

"Je pense qu'il ne faut pas qu'il y ait de journalistes médicaux, je pense qu'il ne faut pas qu'il y ait de journalistes scientifiques et je pense que, dans la mesure où on est dans le grand public, on est journaliste tout court. Et que, finalement, partir en étant un peu vierge sur les contenus compliqués, eh bien! c'est la meilleure garantie à avoir du bon sens et à bien expliquer les choses aux gens" (entretien avec un ancien journaliste spécialisé de la télévision, 1995).

"Les spécialistes du journal nous regardent de haut, nous, la petite escouade de reporters, et nous les méprisons joyeusement. Entre nous les camps sont tracés. Inhibés par la proximité de leurs sources, les spécialistes n'écrivent pas le dixième de ce qu'ils savent; ils se fichent du lecteur; ils n'écrivent que pour les autres spécialistes» (extrait du livre de Daniel Schneidermann, ancien grand reporter au Monde: Du journalisme après Bourdieu, Paris, Fayard, 1999, p. 23).

Face à ces modèles professionnels, les journalistes d'investigation ont contribué à faire émerger une nouvelle figure, celle du justicier. Au nom des demandes ou des attentes de "l'opinion publique", qui serait soucieuse d'une plus grande transparence, ils se posent en concurrents des plus hautes instances judiciaires et politiques. Par exemple, ils instaurent des relations plus critiques avec des sources judiciaires qui, de fait,

39 - E. Plenel, Lntemps de chien, Paris, Stock, 1994, p. 186 40 - Voir D. Marchetti. op. cit. 2' partie, chap. 2 
viennent disqualifier celles qu'entretiennent les chroniqueurs judiciaires. Alors que les premiers « respectent les usages des institutions", les seconds "les mettent en cause " et dénoncent les règles du jeu ${ }^{41}$ en vigueur jusque-là. Certains chroniqueurs judiciaires (mais aussi d'autres " rubricards") ${ }^{42}$ regardent souvent les "investigateurs » comme des journalistes sans scrupules ou sans déontologie parce qu'ils violeraient les règles avec tel ou tel magistrat ou avocat, c'est-à-dire qu'ils seraient prêts à tout pour diffuser une information exclusive : « Je n'ai rien contre le fait qu'on sorte des affaires parce que je crois que c'est bien dans une démocratie, etc. Mais il y a des fois où c'est un peu n'importe quoi...", explique ainsi un reporter-chroniqueur judiciaire d'une radio nationale (entretien, 1998).

La structure du capital de relations professionnelles distingue ces deux catégories de professionnels. Si les journalistes judiciaires ont un carnet d'adresses centré essentiellement sur l'institution judiciaire, autrement dit des magistrats et des avocats, l'administration ou le ministère de la Justice, les seconds, compte tenu des affaires qu'ils traitent, ont des réseaux de relations non seulement dans le secteur judiciaire, mais aussi auprès des enquêteurs dépendant du ministère de l'Intérieur (les policiers, les membres de services de renseignements) ou de la défense (les gendarmes) et des hommes politiques ou de leur entourage.

Toutefois la valorisation de ce nouvel idéal professionnel est un enjeu important pour l'ensemble du champ journalistique, dans la mesure où il a aussi des usages externes, permettant aux journalistes de réaffirmer la légitimité souvent contestée de leur profession et leur autonomie à l'égard des univers sociaux dont ils parlent. La construction par les journalistes de l'affaire du sang contaminé en exemple emblématique le montre particulièrement bien. Aux décisions de justice et aux projets de loi sur la presse qui leur sont défavorables, les journalistes opposent en effet très souvent l'exemple de ce "scandale " qui, selon eux, n'a pu être porté sur la place publique que grâce au travail journalistique. Les enquêtes sur le «scandale du sang contaminé " incarnent un journalisme indépendant des pouvoirs, qui dévoile la vérité que d'autres (journalistes concurrents, médecins de la transfusion, hommes politiques, etc.) tenteraient de minimiser, voire d'étouffer. De nombreux journalistes, qui ont travaillé sur les contaminations post-transfusionnelles, expliquent qu'ils ont largement contribué à faire connaître aux publics de leurs médias respectifs ou plus largement au grand public le drame que vivaient des centaines d'hémophiles et de transfusés ainsi que leur entourage. Ils montrent qu'ils ont fait leur métier en dénonçant des faits délictueux, contraires à une sorte de morale publique, développant parfois l'image forte du journaliste défenseur du "peuple " contre "l'intelligentsia ${ }^{43}$. Selon certains journalistes, l'enquête de L'Événement du jeudi et le travail de quelques journalistes spécialisés ou de reporters généralistes n'auraient pas seulement été à l'origine d'une intense mobilisation médiatique, mais auraient aussi pesé sur la première instruction judiciaire. Certains vont jusqu'à affirmer qu'il n'y aurait pas eu d'affaire judiciaire en l'absence de l'intervention des médias au printemps 1991. Comme dans d'autres affaires, cette interprétation surestime clairement le "pouvoir» de la presse. Il suffit en effet de rappeler que la première instruction avait débuté dès la fin des années 80 .

"L'affaire se serait sans doute éteinte dans un non-lieu si le scandale n'avait éclaté dans la presse ${ }^{+4}$, explique par exemple une journaliste médicale de Libération tandis qu'Anne-Marie Casteret de L'Événement du jeudi se dit convaincue que c'est la "publication de ce document lun rapport du CNTS datant de 1985 qu'elle a "sorti" fin avril 1991] qui a provoqué le tournant judiciaire de cette affaire ${ }^{45}$, assurant, dans une interview ${ }^{46}$ : "Je peux vous dire que si le scandale n'avait pas éclaté [sous-entendu dans la presse], on s'acheminait tout droit vers un non-lieu général ". "Je crois que si je n'avais pas eu ce document, ça ne serait jamais sorti. Ça ne serait jamais sorti » (entretien, 1992). "La presse, relayée par l'opinion publique, a efficacement joué son rôle: sans elle, les hémophiles seraient morts dans le silence. Et sans scandale », explique un journaliste d'InfoMatin (22 septembre 1994). Mark Hunter soutient ainsi que « ce n'est qu'après les premières révélations d'Anne-Marie Casteret dans L'Événement du jeudi que de vraies enquêtes judiciaires et officielles ont été mises en route ${ }^{47}$.

41 - R. Lenoir, "Champ judiciaire et réforme de l'instruction ", art cit., p. 197-198

42 - Un journaliste politique d'un grand quotidien, qui venait de signer un article avec un journaliste d'investigation, expliquait de manière humoristique a un de ses collègues: "Tu as vu, j'ai signé avec un flic!"

43 - "Anne-Marie Casterel fjournaliste spécialisèe à L'Événement du jeudi, qui a publié, le 25 avril 1991, un document prouvant aux yeux des journalistes qu'"on" avait sciemment distribue des produits contaminés\}, en révélant cette affaire, a permis aux victimes de se défendre quand elles le pouvaient encore. Elle leur a également rendu leur dignité. Ces victimes qui, souvent, étaient des enfants et qui, pendant de longues années, n'ont pu que souffrir en silence", explique une journaliste de France Inter (Le Choix d'Inter, 31 janvier 1999). Interviewant Anne-Marie Casteret, un de ses confreres d'Europe 1 (Arrêt sur info, 25 février 1999) note: "Dans cette affaire, vous êtes, et cela vous honore, du côté des plus faibles, des victimes. "

44 - Liberation, 21 septembre 1994

45 - Extrait d'une interview publiee dans N. Pinard et E. Rémond, "Le guide du reporter dans le monde judiciaire", Bordeaux, IUT journalisme de Bordeaux-France 3 Aquitaine, 1995, p. 83-85

46 - Le Quotidien de Paris, 24 février 1992.

47 - M. Hunter. Aspects du journalisme d'investigation. Psychologie, méthodologie, et stratégies narratives de trois enquêtes européennes, incluant une histoire de l'investigation en France et aux Etats-Unis depuis 1973. Paris, universite Panthéon-Assas, Institut français de presse, these en sciences de l'information sous la direction de F. Balle, 1996, p. 26. 


\section{Le contre-exemple de la chronique judiciaire}

A lors que le suivi des instructions des affaires en cours est un exercice hautement concurrentiel, le traitement des procès et de l'information judiciaire institutionnelle l'est beaucoup moins. Les reporters-chroniqueurs judiciaires jouissent d'un certain confort, comme le dit l'un d'entre eux (entretien, 1997), puisqu'ils ont généralement accès aux mêmes informations, et en même temps, le cas des procès illustrant particulièrement bien ce type de situation où le scoop n'est pas un enjeu. Si l'information dans ce domaine peut être parfois diffusée en exclusivité par un média, elle l'est par le biais des agences de presse qui la transmettent de fait à l'ensemble des supports abonnés. Le microcosme des reporters-chroniqueurs judiciaires fonctionnant comme " une petite famille $)^{\mathrm{a}}$ qui est «accréditée » par l'institution n'est donc pas concurrentiel et très peu conflictuel. L'entraide est permanente dans les discussions, particulièrement lors des procès, où les reporters-chroniqueurs communiquent leurs impressions, échangent des sons et des images, rapportent des propos parce que l'un d'entre eux était en train de faire son papier ou qu'il avait un problème technique par exemple. Si des sous-groupes existent, ils se retrouvent très souvent sur les mêmes lieux, qu'il s'agisse de la salle qui leur est allouée au sein du Palais de justice de Paris (essentiellement occupée par les agenciers), des couloirs ou de la buvette du Palais, des salles d'audience et, plus encore, des hôtels et des restaurants lorsqu'ils couvrent des procès en dehors de la région parisienne. Ils sont tous regroupés au sein d'une association spécialisée, qui gère notamment les accréditations lors des procès et les relations avec les institutions judiciaires, mais joue aussi le rôle d'instance de socialisation professionnelle, où les «anciens parrainent» les nouveaux entrants. Cette forme d'autorégulation peut aller jusqu'à l'entraide spontanée, comme en témoigne l'exemple suivant : « II peut nous arriver, on entend un confrère dicter son papier et puis y a un mot, pas le bon, aussitôt, on lui dit: "Non" [il fait des gestes négatifs avec la main]. "Ne quittez pas" [il mime toujours]. "Quoi ça va pas." "Non là, c'est pas juste, c'est l'article tant c'est..." "Ah bon!" Alors qu'on travaille pour des organismes différents hein!» (entretien, 1995).

a - Entretien, 1998.
Le "scandale du sang contaminé " ne serait sans doute pas devenu une affaire aussi exemplaire s'il n'était pas apparu dans la presse en 1991, c'est-à-dire peu de temps après la révolution roumaine et la guerre du Golfe. Il fournissait en effet un contre-exemple susceptible d'être opposé aux critiques qui, au sein de la profession et en dehors, mettaient en cause l'indépendance, voire la moralité des journalistes en s'appuyant sur les « dérapages» et les «dérives » auxquels ces deux événements avaient donné lieu. Sujets quasi obligés dans les livres, les articles ou les colloques sur le journalisme, la révolution roumaine et la guerre du Golfe symbolisaient cette "perte de crédit " de la profession que le sondage commandé à la Sofres en novembre 1991 par Médiaspouvoirs, La Croix et Télérama est censé mesurer $^{+8}$. Le "scandale du sang contaminé " permettait en quelque sorte de laver "l'honneur de la profession».

\section{Les effets sur la production de l'information}

L'imposition de ce nouveau modèle d'excellence professionnelle et la constitution de ces nouvelles positions dans le champ journalistique ont eu notamment des effets sur le traitement de trois grands secteurs d'activité (politique, police et justice). En fait, les journalistes d'investigation sont venus concurrencer plu- sieurs genres journalistiques traditionnels. En venant sur le terrain de l'information politique, les journalistes-enquêteurs ont contribué à " couvrir » l'actualité politique sous forme de faits divers, technique qui avait été développée mutatis mutandis par la presse populaire à la fin du $\mathrm{xIX}^{\mathrm{C}}$ siècle ${ }^{+9}$. S'inscrivant en rupture avec les éditorialistes politiques et les dirigeants des grands médias, ils se séparent de la tradition littéraire et politique de la presse française en prônant une autre conception de l'information politique: le journalisme d'enquête qui dévoile des "faits cachés" s'oppose au journalisme d'opinion, à celui qui privilégie le commentaire, les idées, le parti pris (qui est ou était autorisé pour les "critiques " littéraires et cinématographiques notamment) au détriment des « faits».

Les journalistes d'investigation ont également participé à une redéfinition de ce que recouvrent les faits divers et la chronique judiciaire. Sils se réclament de la tradition du fait divers ${ }^{50}$ et même ont eu pour

48 - Télerama, 18 et 24 janvier 1992

t9 - M. Palmer. Des petits journaux aux grandes agences. Naissance du foumalisme moderne, Paris, Aubier, 1983, p. 259.

50 - Sur ce sujet, voir D. Kalifa. "Les tâcherons de l'information petits reporters et faits divers a la "Belle Epoque" ". in Re'rut d'histome moderne it contemporane vol 40, $\mathrm{n}^{\circ}+.1993, \mathrm{p}$. 578-603: Lincre et le sang. Recits de crimes et sedele a la Belle Epoque. Paris. Fayard, 1995 ; et 11. Lever. Canards sanglants Nuissanci du fatt divers, Paris. Fayard, 1993. 
beaucoup d'entre eux à traiter de cette matière au début de leur carrière ${ }^{51}$, les journalistes d'investigation, en portant davantage leur attention sur des faits à caractère politique, qui impliquent des personnalités connues ou engagent l'État, ont cherché à anoblir professionnellement cette rubrique. Une des conditions d'accès à la reconnaissance professionnelle était de politiser le fait divers: "S'intéresser à je ne sais quelle corruption est peut-être un peu plus noble que d'aller ennuyer les parents d'une jeune fille violée ", ironise un chroniqueur judiciaire d'un quotidien national (entretien, 1995).

L'arrivée des journalistes d'investigation a enfin contribué au déplacement progressif du centre de gra$v$ vité $^{52}$ de la médiatisation de la vie judiciaire du procès vers l'instruction, faisant perdre à la chronique judiciaire une partie de son prestige. Considérée comme une des rubriques nobles du journalisme français qui était tenue par des journalistes expérimentés, loués pour leur plume, voire par des écrivains de renom ${ }^{53}$ après la guerre, elle avait déjà évolué puisque la médiatisation des activités judiciaires, qui concernait essentiellement la couverture des procès (essentiellement d'assises), s'était élargie dans les années 70 au fonctionnement même de l'activité judiciaire institutionnelle dans des nouvelles rubriques intitulées "Vie judiciaire », "Justice », etc. ${ }^{54}$. Aujourd'hui, l'essentiel de l'actualité judiciaire porte sur les annonces des mises en examen, le déroulement de perquisitions, l'audition de protagonistes par le magistrat instructeur, qui donnent lieu à des comptes rendus immédiats. Si bien que seules les rédactions les plus nombreuses (les agences de presse comme l'AFP et Reuter, TF1, France 2 et France 3, Europe 1, France Inter, Le Monde, Le Figaro notamment) ont encore des chroniqueurs judiciaires attitrés. La place accordée aux procès est moindre, les audiences sont de moins en moins nombreuses à être suivies et ce sont aussi désormais des reporters ou des grands reporters des services d'information générale, voire d'autres journalistes spécialisés qui les couvrent.
51 - Pour un exemple, voir J. Derogy et J.-M. Pontaut, op. cit., p. 7. 52 - R. Lenoir, "Champ judiciaire et réforme de l'instruction ", in M. Delmas-Marty (sous la dir. de), Procès pénal ét droits de l'homme. Vers une conscience européenne, Paris, PUF, 1992, p. 197.

53 - Voir le témoignage de J.-M. Théolleyre, ancien chroniqueur judiciaire au Monde, dans N. Pinard et E. Rémond, op. cit., p. 63

$54-\mathrm{R}$. Lenoir et $\mathrm{S}$. Poilleux, Justice et médias. Le secret de l'instruction et le droit au respect de la présomption d'innocence, op. cit., p. 69. 